NOTE

\title{
Synthesis of Star Polymer with Nucleus of Microgel
}

\author{
Koji Ishizu, Shinichi Gamoo, Takashi FukUtomi, \\ and Toshio KAKURAI
}

Department of Polymer Technology, Tokyo Institute of Technology, 2-12, Ookayama, Meguro-ku, Tokyo 152, Japan.

(Received August 30, 1979)

\begin{abstract}
KEY WORDS Microgel / Ultrasonic Irradiation / Vinyl Group / Butyl Lithium / Polyanion / Star Polymer /
\end{abstract}

Work on star polymers has revealed some important properties which differ with those of linear polymers. It can be expected that the density and refractive index of a branched polymer are larger than those of the linear polymer, and the intermolecular interaction and coefficient of thermal expansion, are smaller. Recently, it has been found that anionic propagation and coupling methods can be used to the preparation of well-defined star polymers. ${ }^{1-10}$ Thus far, two proven methods have been used: (1) the reaction of a polyfunctional electrophile species with monocarbanionic chains, ${ }^{1-6}$ and (2) the synthesis of star-polymer materials wherein the active-chain ends of highmolecular chains are reacted with divinylbenzene (DVB) as a linking agent. ${ }^{7-10}$ On the other hand, the cross-linking reaction of styrene-DVB is well known to produce macrogel and microgel by changing the reaction conditions, i.e., the monomer concentration in the polymerization system and the feed ratio of styrene and DVB.${ }^{11}$ Our laboratory has also reported the results which show that the ultrasonic irradiation in the radical polymerization of styrene-DVB yielded microgels having high conversion and a rich content of intramolecular cross-linking. ${ }^{12}$ This microgel has some vinyl groups and the syntheses of star polymers having morecomplicated molecular structure can possibly be obtained by using it.

\section{EXPERIMENTAL}

The microgel was synthesized in the previous way. ${ }^{12}$ In short, the radical polymerization of styrene-DVB was carried out in dioxane at $60^{\circ} \mathrm{C}$ by redox system (benzoyl peroxide-dimethylaniline) as a initiator under ultrasonic irradiation. The ultrasonic irradiation was carried out with a Cho-Onpa Kogyo Co., Ltd. UH-3C at a frequency of $25 \mathrm{KHz}$, using a nickel transducer under a nitrogen atmosphere. Polymerization was stopped by introducing the resulting viscous solution into excess methanol. The microgel was separated as a precipitate. The reaction of butyl lithium (BuLi) with the residual vinyl groups in the microgel was carried out in benzene for $48 \mathrm{~h}$ at $30^{\circ} \mathrm{C}$ under the following conditions: vinyl groups, $6.0 \times 10^{-3} \mathrm{moll}^{-1}$ and $\mathrm{BuLi}, 0.16 \mathrm{moll}^{-1}$ (it was found by analysis with ultraviolet spectra that the residual vinyl groups in the microgel reacted completely with BuLi after a few hours under the same reaction conditions described above). Separation of the polyanion from the mixture solution obtained above was carried out by the following procedure. Heptane is a nonsolvent for polystyrene and dissolves BuLi. The boiling point of heptane is higher about $20^{\circ} \mathrm{C}$ than that of benzene. As shown in Figure 1, the apparatus was sealed off from the vacuum line and washed with a sodium naphthalene solution (D), which was later recovered into $C$ and sealed off. First, the metallated solution (B) was transferred to $\mathrm{E}$ and heptane (A) was gradually dropped into $\mathrm{E}$ (benzene/heptane $=1 \mathrm{v} / 1 \mathrm{v}$ ). Next, benzene was distilled from $\mathrm{E}$ to $\mathrm{F}$ until the polyanion precipitated. The supernatant solution in $\mathrm{E}$ was decanted to $\mathrm{F}$, and the solvent (benzene + heptane) was distilled again from $F$ to $E$. These steps were repeated six 


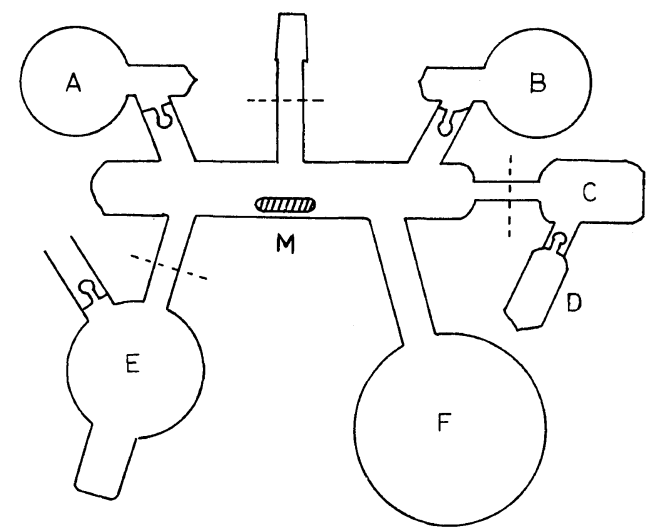

Figure 1. Apparatus to separate polyanion: A, heptane; B, metallated solution; C, E, and F, ampoules; D, solution of sodium naphthalene; $\mathrm{M}$, magnet.

times. Finally, ampoule E was sealed off. The star polymer was prepared by the anionic polymerization of $\alpha$-methylstyrene (MS) with the separated polyanion as an initiation species in tetrahydrofuran (THF) at $-78^{\circ} \mathrm{C}$ by using living method. After $48 \mathrm{~h}$, the polymerization was stopped by coupling the active ends with 1-chloromethylnaphthalene in order to determine the number of branches. The reaction solution was poured into large excess methanol. The precipitate was carefully washed with fresh methanol and dried in vacuo. The microgel and star polymer were characterized by various methods: osmotic pressure measurement in toluene at $37^{\circ} \mathrm{C}$ with a Mechrolab Model 501 Membrane Osmometer, gel-permeation chromatcgraphs (GPC) data with a Toyo Soda High Speed Liquid Chromatograph HLC-801A in THF at $40^{\circ} \mathrm{C}$ with a mixed column having a flow rate of 1.4 $\mathrm{ml} \mathrm{min}^{-1}$, intrinsic viscosity $[\eta]$ in toluene at $25^{\circ} \mathrm{C}$ with a Ubbelohde viscometer. The contents of the vinyl groups in the microgels were measured by a Hitachi Spectrophotometer Model 124 at $292 \mathrm{~nm}$ using $p$-methylstyrene as a reference in chloroform, and the contents of naphthalene groups in polymers were also measured by the same instrument at $315 \mathrm{~nm}$, using poly(MS) $\left(M_{n}=24000\right)$ which has a naphthalene group at the end, as a reference.

\section{RESULTS AND DISCUSSION}

The results of microgel syntheses were shown in Table I. The purity of DVB used in this work was $55 \%$ and was a mixture of $m$-DVB and $p$-DVB. The ratio of $m$-DVB $/ p$-DVB was 2.5 from gas chromatography. Malinsky and coworkers ${ }^{14}$ reported the reactivity ratios of the free-radical copolymerization of styrene $\left(\mathrm{M}_{1}\right)$ at $70^{\circ} \mathrm{C}$ to be $r_{1}=0.54, r_{2}=0.58$ for $m$-DVB $\left(\mathrm{M}_{2}\right)$; and $r_{1}=0.15, r_{2}=1.22$ for $p$-DVB $\left(\mathrm{M}_{2}\right)$, indicating that the vinyl group is more reactive in $p$-DVB than the vinyl group in $m$-DVB. Considering that the conversion of the reaction product was $3-9 \%$, only $p$-DVB contributed to

Table I. Results of microgel syntheses ${ }^{\mathrm{a}}$

\begin{tabular}{|c|c|c|c|c|c|c|c|c|c|}
\hline \multirow{3}{*}{ No. } & \multirow{3}{*}{$\frac{\text { Styrene }}{\mathrm{ml}}$} & \multirow{3}{*}{$\frac{\text { DVB }}{\mathrm{ml}}$} & \multirow{3}{*}{$\frac{\text { Yield }}{\mathrm{g}}$} & \multirow{3}{*}{$\frac{M_{n}}{\times 10^{-4}}$} & \multicolumn{4}{|c|}{ Vinyl groups } & \multirow{3}{*}{$\frac{\begin{array}{c}{[\eta]^{\mathrm{d}}} \\
(\text { linear) }\end{array}}{\mathrm{dl} \mathrm{g}^{-1}}$} \\
\hline & & & & & \multicolumn{2}{|c|}{ Number/molecule } & \multicolumn{2}{|c|}{$\begin{array}{c}\text { Number/styrene } \\
100 \text { units }\end{array}$} & \\
\hline & & & & & Found $^{\mathrm{b}}$ & Calcd $^{\mathrm{c}}$ & Found $^{\mathrm{b}}$ & Calcd $^{\mathrm{c}}$ & \\
\hline G1 & 40 & 5 & 3.9 & 5.7 & 23 & 47 & 4 & 10 & $\begin{array}{c}0.26 \\
(0.31)\end{array}$ \\
\hline $\mathrm{G} 2$ & 40 & 10 & 2.3 & 3.8 & 30 & 54 & 8 & 18 & $\begin{array}{c}0.16 \\
(0.25)\end{array}$ \\
\hline G3 & 40 & 15 & 1.3 & 4.8 & 44 & 89 & 10 & 25 & $\begin{array}{c}0.14 \\
(0.29)\end{array}$ \\
\hline
\end{tabular}

a Solvent, dioxane $100 \mathrm{ml}$ : initiator, benzoyl peroxide $0.30 \mathrm{~g}$ and dimethyl aniline $0.2 \mathrm{ml}$; reaction time, $300 \mathrm{~min}$.

b Measured by UV spectra.

c Calculated from the reactivity ratio of the radical copolymerization of styrene and $p$-DVB.

d Measured in toluene at $25^{\circ} \mathrm{C}$.

e $[\eta]$ of linear polystyrene having the same molecular weight calculated by the following equation. $[\eta]=1.7 \times 10^{-4}$ $M_{w}{ }^{0.69}$ in toluene at $25^{\circ} \mathrm{C} .^{13}$ 
these copolymerizations. Based on this data, the contents of vinyl groups introduced into microgel molecule was calculated theoretically, neglecting the effect of ultrasonic irradiation (see Calcd in Table I). Thus, the degree of intramolecular cross-linking is equal to [ [(vinyl groups) $)_{\text {Calcd }}$-(vinyl groups) ${ }_{\text {Found }}$ ]. From these results, the number of vinyl groups and cross-linking density per styrene 100 units increased with an increment in the ratio of DVB to styrene. [ $\eta]$ of each reaction product was smaller than that of linear polystyrene having the same molecular weight. It was found that these reaction products were microgels having high intramolecular crosslinking densities in the order, G3, G2, and G1. GPC profiles of $\mathrm{G} 1-\mathrm{G} 3$ are shown in Figure 2. The GPC

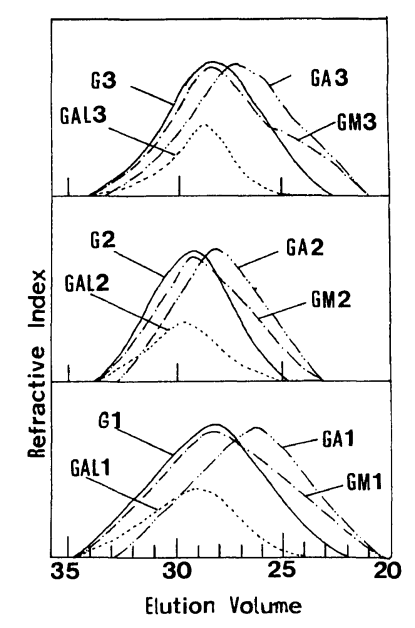

Figure 2. GPC profiles of $G, G M$, and GA series:

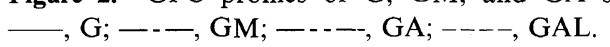

profiles of the reaction products of $\mathrm{BuLi}$ with residual vinyl groups in the DVB microgels (GM series) and those after purification operations (GA series) are also summurized in Figure 2. Characterization of the purified polyanions is shown in Table II. Each GM GPC was shifted to the high-molecular-weight side, compared to that of original microgel. It is considered that the oligomerization of microgel itself is carried out during the reaction of microgel with BuLi. On the other hand, all GA GPC were cut off from the low-molecularweight (GAL) part, but GM GPC were not cut off, because of the fractional precipitation by the purification operation. The yields of GA microgels (GA1-GA3) were about $60 \mathrm{wt} \%$. The GPC profiles of the GAL series are also shown in Figure 2. From the results in Tables I and II, it can be seen that 1-chloromethylnaphthalene couples quantitatively with polyanion.

The anionic polymerizations of MS were carried out in THF solvent, using these polyanions (GA1GA3) as initiation species. The results of the polymerization of MS are shown in Table III and Figures $3 a-3 c$. From Figures $3 a-3 c$, the GPC of each product (GAP1 - GAP3) shows a single peak more narrow than that of the respective polyanion

Table II. Characterization of purified polyanion

\begin{tabular}{lccc}
\hline & GA1 & GA2 & GA3 \\
\hline $\begin{array}{l}M_{n} \times 10^{-4} \\
\begin{array}{l}\text { Anion number/ } \\
\text { molecule }\end{array}\end{array}$ & 9.8 & 8.1 & 12.9 \\
\hline
\end{tabular}

a Measured according to the contents of naphthalene groups.

Table III. Results of polymerization of $\mathrm{MS}^{\mathrm{a}}$

\begin{tabular}{|c|c|c|c|c|}
\hline \multirow{2}{*}{ No. } & \multicolumn{2}{|c|}{ Feed concn $/ \mathrm{mol} \mathrm{1}^{-1}$} & \multirow{2}{*}{$\begin{array}{c}M_{n} \text { of } \\
\text { branches }^{b} \\
\times 10^{-4}\end{array}$} & \multirow{2}{*}{$\frac{\text { Yield }}{\mathrm{g}(\%)^{\mathrm{c}}}$} \\
\hline & Polyanion initiator, $[\mathrm{I}]_{0}$ & MS, $[\mathrm{M}]_{0}$ & & \\
\hline GAP1 & $5.2 \times 10^{-3}$ & 0.7 & 1.58 & $5.1(95)$ \\
\hline GAP2 & $1.8 \times 10^{-2}$ & 1.5 & 0.81 & $3.5(100)$ \\
\hline GAP3 & $3.2 \times 10^{-2}$ & 2.2 & 0.98 & $11.5(98)$ \\
\hline
\end{tabular}

a Solvent, THF; reaction temp, $-78^{\circ} \mathrm{C}$; reaction time, $48 \mathrm{~h}$.

b Calculated from feed concentrations: $\left([\mathrm{M}]_{0} /[\mathrm{I}]_{0}\right) \times 118$.

c Anionic polymerization of MS was carried out quantitatively. 
Table IV. Characterization of each fraction from sample GAP1-GAP3

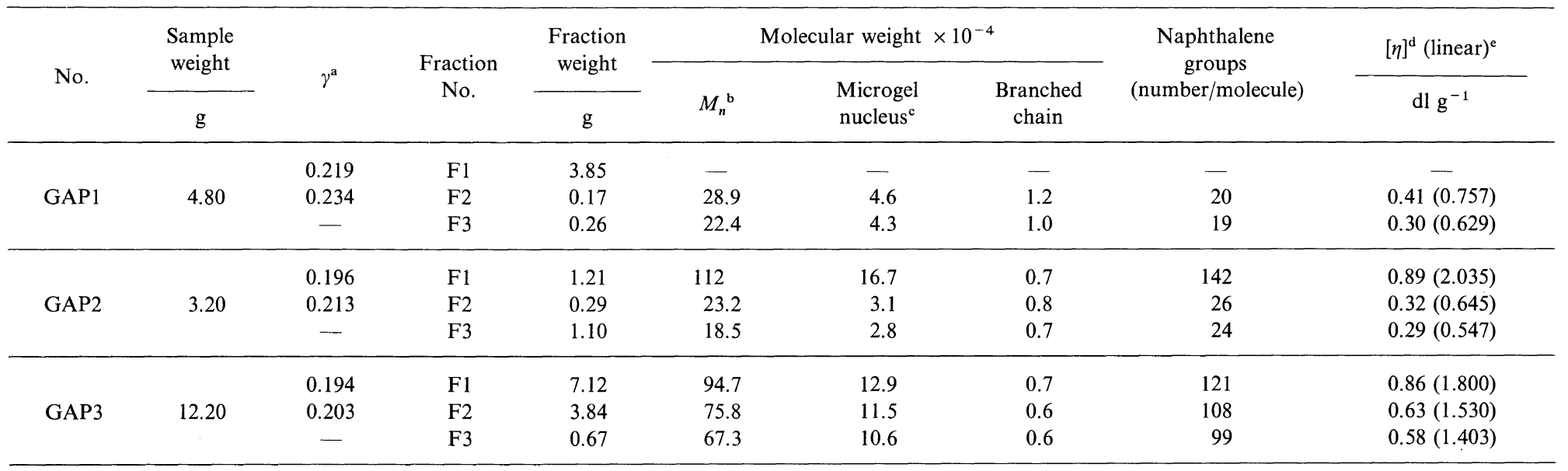

a Value $(\mathrm{v} / \mathrm{v})$ of $\mathrm{MeOH} /(\mathrm{MeOH}+$ benzene $)$.

b Measured by osmometry.

${ }^{c}$ Calculated on the assumption that the distribution of anion density is constant in spite of the molecular weight of the purified polyanion nucleus.

d Measured in toluene at $25^{\circ} \mathrm{C}$.

${ }^{\mathrm{e}}[\eta]$ of linear poly(MS) having the same molecular weight calculated by following equation. $[\eta]=0.781 \times 10^{-4} M_{w}{ }^{0.73}$ in toluene at $25^{\circ} \mathrm{C}^{15}$ 


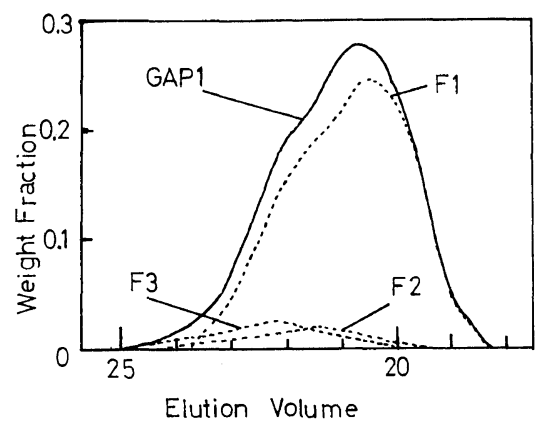

Figure 3a. GPC profiles of GAP1 and each fraction.

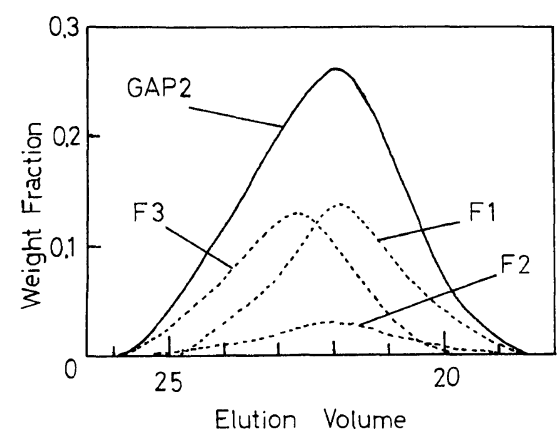

Figure 3b. GPC profiles of GAP2 and each fraction.

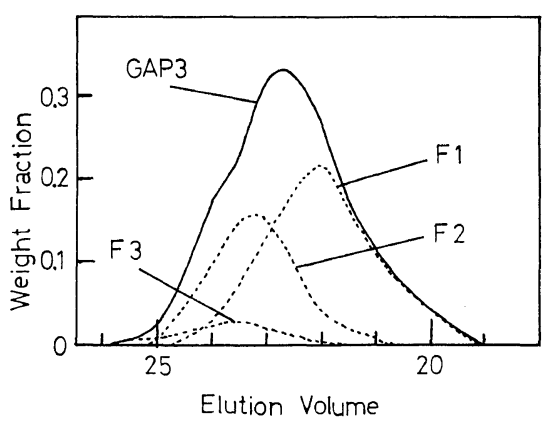

Figure 3c. GPC profiles of GAP3 and each fraction.

nucleus; also, the removal of the BuLi unreacted is performed almost completely in the separation process of polyanion. In order to make clear whether or not the product is the mixture of star polymer and MS homopolymer, and obtained star polymers having a different number of branches, the precipitation fractionation of the products was carried out in a benzene-methanol system at $25^{\circ} \mathrm{C}$ (Table IV). The GPC profile for each fraction is shown in Figures $3 a-3 c$. In the cases of GAP2 and GAP3, the sum of naphthalene contents of the three fractions were nearly equal to those of polyanion nuclei (GA2 and GA3). If linear poly(MS) is contained in GAP, each naphthalene group value for F3 must be about 1. It was found from these results that $\mathrm{GA} 1-\mathrm{GA} 3$ were free from the residual $\mathrm{BuLi}$. The number-average molecular weights of the branched chains of the fractions $(\mathrm{F} 1-\mathrm{F} 3)$ for GAP1-GAP3 were about equal. The $M_{n}$ of the branches nearly agreed with the estimated values from feed concentrations (see Table III). Since MS monomers polymerize in the same manner as equilibrium polymerization, all active species of polyanion nuclei are considered to have a high efficiency of initiation. Also, all initiating species are considered to have the same reactivity in propagation. Thus, the molecular weight distribution of a MS branch is shown to be nearly monodisperse. $[\eta]$ of each branched material is smaller than that of linear poly(MS) having the same molecular weight.

As a result of this study, the branched polymers obtained show characteristics of high compactivity and small intermolecular interaction. It is possible to increase the number of branches by this method. The solution and solid properties of star polymers having microgel nuclei will be reported in the near future.

\section{REFERENCES}

1. M. Morton, T. E. Helminiak, S. D. Gadkary, and F. Bueche, J. Polym. Sci., 57, 471 (1962).

2. T. Altares, Jr., D. P. Wyman, V. R. Allen, and K. Meyersen, J. Polym. Sci., A, 3, 4131 (1965).

3. S. P. S. Yen, Makromol. Chem., 81, 152 (1965).

4. J. E. L. Roovers and S. Bywater, Macromolecules, 5, 385 (1972).

5. L. J. Fetters and M. Morton, Macromolecules, 7, 552 (1974).

6. K. Ishizu, T. Fukutomi, and T. Kakurai, Polym. J., 7, 228 (1975).

7. J. G. Zilliox, Makromol. Chem., 156, 121 (1972).

8. J. G. Zilliox, P. Rempp, and J. Parrod, J. Polym. Sci., C, 22, 145 (1968).

9. L. K. Bi and L. J. Fetters, Macromolecules, 8, 90 (1975).

10. L. K. Bi and L. J. Fetters, Macromolecules, 9, 732 (1976). 


\section{K. Ishizu, S. Gamoo, T. Fukutomi, and T. KaKurai}

11. M. R. Ambler and D. McIntyre, J. Appl. Polym. Sci., 21, 2269 (1977).

12. T. Fukutomi and T. Kakurai, Polym. Prepr., Jpn., 26 (1), 110 (1977).

13. P. Outer, C. I. Carr, and B. H. Zimm, J. Chem. Phys.,
18, $830(1950)$

14. J. Malinsky, J. Klaban, and K. Dusck, J. Macromol. Sci., Chem., A5, 1071 (1971).

15. T. Fujimoto, N. Ozaki, and M. Nagasawa, J. Polym. Sci., A, 3, 2259 (1965). 